\title{
Extending the Depth of Field in a Compound-Eye Imaging System with Super-Resolution Reconstruction
}

\author{
Wai-San Chan and Edmund Y. Lam \\ Department of Electrical and Electronic Engineering \\ The University of Hong Kong \\ Pokfulam Road, Hong Kong \\ wschan@eee.hku.hk and elam@eee.hku.hk
}

\author{
Michael K. Ng \\ Department of Mathematics \\ Hong Kong Baptist University \\ Kowloon Tong, Hong Kong \\ mng@math.hkbu.edu.hk
}

\begin{abstract}
Optical device miniaturization is highly desirable in many applications. Direct down-scaling of traditional imaging system is one approach, but the extent to which it can be minimized is limited by the effect of diffraction. Compound-eye imaging system, which utilizes multiple microlenses in image capture is a promising alternative. In this paper, we explore the possibility of an incorporation of phase masks in such a system to extend the depth of field. Simulation experiments are conducted to verify the feasibility of the system.
\end{abstract}

\section{Introduction}

Many applications call for the miniaturization of optical devices. These include consumer electronic products and also biomedical instrumentations. Direct down-scaling of traditional imaging system is one approach, but the extent to which it can be minimized is limited by the effect of diffraction. Recently developed compound-eye imaging system is considered a promising alternative in this setting. While traditional imaging system uses a single optical channel formed by a set of cascading lenses with large diameters, compound-eye imaging system utilizes an array of multiple microlenses in image capture. Multiple low-resolution (LR) sub-images generated are used to reconstruct a final high-resolution (HR) image. An important motivation of this novel design of imaging system lies in the resulting system compactness.

Compound-eye imaging system design has received a lot of attention in recent years. Besides compactness, other advantages of this design includes lightness and a wide field of view. Several research groups have designed and constructed a variety of compound-eye imaging systems $[3,4,10]$. Multiple optical channels allow greater flex- ibility in the system. One possibility is to add phase masks behind the lenses. A phase mask is a special-shape lens which alters the received incoherent wave front in a specific way. With appropriate restoration, depth of field can be extended [2]. In this paper, we present a compound-eye imaging system with super-resolution reconstruction, and explore the possibility of an incorporation of phase masks in such a system to extend the depth of field. Simulation experiments are also conducted to investigate the performance of our methods. The structure of the paper is as follows: In section 2, we describe the architecture of the compoundeye imaging system of our concern. In section 3, the system model and the super-resolution reconstruction algorithm is explained. Simulation results which investigate the performance of the phase mask-incorporated system are discussed in section 4. Finally, concluding remarks are provided in section 5 .

\section{Architecture of the compound-eye imaging system}

Compound-eye imaging system takes into account the optics, optoelectronic, and image processing as a whole in the design. In a compound-eye system, multiple microlenses converge light onto the optoelectronic components. The optical information collected is processed in one way and another in an attempt to restore a fine image of the target object. Fig. 1 shows the architecture of the baseline system that we are considering in this paper [10]. The system consists of an $N \times N$ microlens array and a photodetector array with $M \times M$ photosensitive cells. Thus, each microlens corresponds to a sub-array of $\alpha \times \alpha$ cells, where $\alpha=\frac{M}{N}$. Each microlens together with its array of photosensitive cells form an imaging unit, which generates a LR sub-image (with resolution $\alpha \times \alpha$ ) of the object. A separation layer is placed between the microlens array and the photodetector array to minimize crosstalk among sub- 


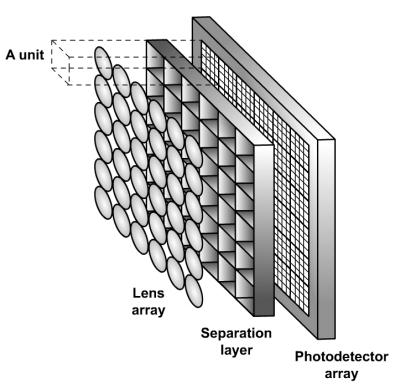

\section{Figure 1. Architecture of the compound-eye system [10]}

images.

The sampling method [10] and pixel rearrangement [7] are image reconstruction methods proposed for this compound-eye system. The latter method shows a significant improvement over the former, but still there is room for improvement in terms of image quality. Indeed, provided that there are sub-pixel displacements in all the subimages so that there are no redundant sub-images, superresolution can be employed to reconstruct a HR image from the multiple LR sub-images [1]. The working principle of the compound-eye imaging system with super-resolution reconstruction is shown in Fig.2.

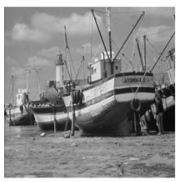

Target Object
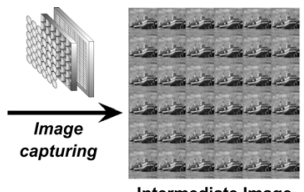

Intermediate Image

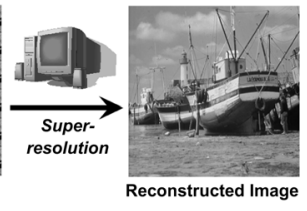

Figure 2. Flow diagram of the compound-eye imaging system.

\section{System modeling, phase masks incorpora- tion, and super-resolution reconstruction}

In this section, we describe the mathematical modeling of a phase mask-incorporated compound-eye system, and the super-resolution algorithm we used in the reconstruction process. Assuming linearity in the entire electro-optical system, we use matrix equations in describing the effects of various components [5]. All the images are represented by vectors formed by lexicographical ordering of the image pixels. The target object is regarded as the HR image on which a series of operations are performed to produce the LR sub-images. Since each imaging unit operates individually in its own optical path, under the assumption that there is no crosstalk between the sub-images, the formation of each sub-image can be modeled independently from one another in its own channel. Consequently, a compound-eye system with $N \times N$ lenses gives $N^{2}$ optical channels and thus $N^{2}$ sub-images.

Consider the modeling of the $k$ th optical channel. The corresponding $k$ th sub-image $\mathbf{i}_{k}$ is of length $\alpha^{2}$. Firstly, as the $k$ th lens is displaced vertically and horizontally from a reference lens, the $k$ th sub-image formed is shifted away correspondingly from a reference frame. This is modeled by the shifting matrix $S_{k}$. Secondly, the lens introduce blurring to the sub-images. This is modeled by the blurring matrix $B_{k}$. Thirdly, the phase mask introduces a path length error to the sub-image. This is represented by the phase mask operator $M_{k}$. Fourthly, the finite-size photosensitive cells further blur and downsample the sub-image. This is represented by the down-sampling matrix $D_{k}$. Fifthly, noise is added to the sub-image due to noise of the photosensitive cells and quantization. This is represented by the noise vector $\mathbf{v}_{\mathbf{k}}$. Finally, the sub-image is passed through a restoration filter $F_{k}$ which restore the sub-image. Mathematically, we have the matrix equation

$$
\mathbf{i}_{\mathbf{k}}=F_{k}\left(D_{k} M_{k} B_{k} S_{k} \mathbf{f}+\mathbf{v}_{\mathbf{k}}\right) .
$$

Stacking all the $N^{2}$ equations for the $N^{2}$ channels results in a single matrix equation given by

$$
\mathbf{i}=H \mathbf{f}+\mathbf{v}
$$

where $H$ is called the blurring matrix of the whole system, and $\mathbf{v}$ is the noise vector. Fig. 3 shows schematically the phase mask-incorporated system model. Since solving the

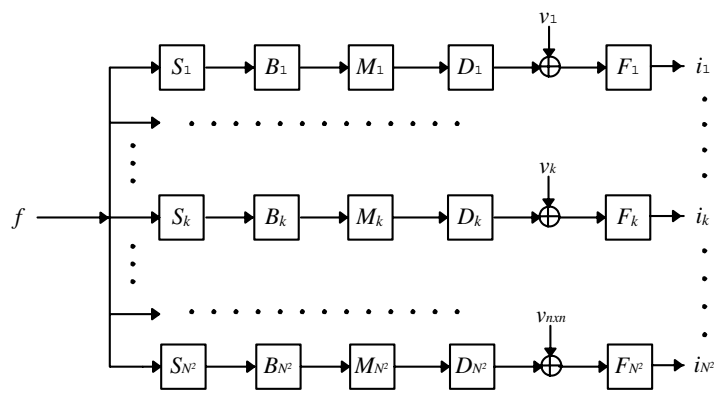

Figure 3. Schematic diagram of the phase mask-incorporated compound-eye imaging system.

high-resolution image $\mathbf{f}$ from the matrix equation is an illconditioned problem, the minimization and regularization techniques are used [1]:

$$
\min _{\mathbf{f}}\|H \mathbf{f}-\mathbf{i}\|^{2}+\beta \mathcal{R}(\mathbf{f})
$$


where the function $\mathcal{R}(\mathbf{f})$ measures the regularity of $\mathbf{f}$ and $\beta$ is the regularization parameter which controls the regularity of the solution. Using the Tikhonov regularization, the problem becomes

$$
\left(H^{T} H+\beta R\right) \mathbf{f}=H^{T} \mathbf{i}
$$

where $R$ is the discretization matrix corresponding to the regularization functional $\mathcal{R}(\mathbf{f})$ [8]. The linear system can be solved iteratively by the conjugate gradient method. Cosine transform preconditioners are used to increase the computational speed for the reconstruction process. Details can be found in [9].

\section{Simulation}

A virtual camera is built to investigate the capability of a compound-eye system with an incorporation of phase masks to extend the depth of field. The virtual camera can simulate an $N \times N$ compound-eye system where $N$ can take on any positive integer value. Different types of phase masks can be inserted into the system. The virtual camera takes into account the effect of diffraction on the images produced. For a diffraction limited system, the point-spread function (PSF) of an in-focus image is given by the Fraunhofer diffraction pattern of the lens aperture. This is given by

$$
\begin{aligned}
h(u, v)= & \frac{A}{\lambda z_{i}} \int_{-\infty}^{\infty} \int_{-\infty}^{\infty} P(x, y) \\
& \exp \left\{-j \frac{2 \pi}{\lambda z_{i}}(u x+v y)\right\} \mathrm{d} x \mathrm{~d} y
\end{aligned}
$$

where the pupil function $P$ is unity inside and zero outside the projected aperture, $A$ is a constant amplitude, $z_{i}$ is the image distance, $\lambda$ is the wavelength of the illumination, and $(x, y)$ are coordinates in the plane of the exit pupil. We can see that the effect of diffraction is actually realized by convolving the object with the Fraunhofer diffraction patter of the lens aperture, which leads to a loss of fine details of the object [6].

We investigate the use of phase masks together with restoration filters in extending the depth of field. Simulations have been conducted using the virtual camera together with the super-resolution reconstruction algorithm. For simplicity, a compound-eye system with $2 \times 2$ imaging units is simulated, and the following assumptions are made: (1) there is no misalignment of the lens and photodetector arrays; (2) the relative shifts between the sub-images are exactly $\frac{1}{2}$ pixel-width; (3) there is no crosstalk between the sub-images, and (4) the four lenses all have the exact required focal length and have no aberrations. In our experiments, cubic phase masks are used [2]. When we placed a cubic phase mask behind a lens, we are deliberately introducing a path length error which is given by

$$
W(x, y)=\exp \left\{j \frac{\gamma}{R^{3}}\left(x^{3}+y^{3}\right)\right\} .
$$

where $\gamma$ is a constant which controls the phase deviation. Wiener Filter is used as the restoration filter in our experiments. It is given by

$$
G\left(f_{X}, f_{Y}\right)=\frac{\mathcal{H}^{*}\left(f_{X}, f_{Y}\right)}{\left|\mathcal{H}\left(f_{X}, f_{Y}\right)\right|^{2}+\frac{S_{v}\left(f_{X}, f_{Y}\right)}{S_{i}\left(f_{X}, f_{Y}\right)}}
$$

in the frequency domain where $\mathcal{H}$ is the OTF of the individual optical channel, $S_{i}\left(f_{X}, f_{Y}\right)$ and $S_{v}\left(f_{X}, f_{Y}\right)$ are the power spectra of the undegraded image and noise respectively. In our simulated compound-eye system, there are four optical channels. Therefore, four cubic phase masks are incorporated into the system, with each lens being followed by one cubic phase mask. Fig.4 compares the reconstructed images obtained from a system without using cubic phase mask and a system with an incorporation of four phase masks after restoration by Wiener filters. (In the scene, the cup is placed at the ideal object distance, while the book is placed at distances which is $20 \%$ shorter than the ideal object distance.) It is clear that while some parts of the reconstructed images obtained from the system without phase masks are out-of-focus, the phase masksincorporated system can bring the whole scene with different depths into focus. This demonstrates the capability of cubic phase masks in extending the depth-of-field in a compound-eye system .

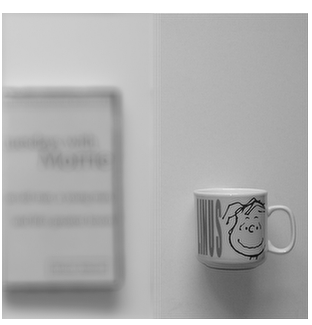

(a) Without phase mask.

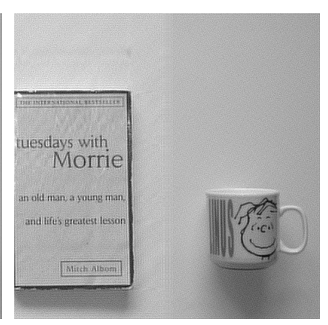

(b) With phase masks.

\section{Figure 4. Reconstructed images obtained from a scene with different depths.}

Since there are multiple optical channels in a compoundeye system, there is a possibility of incorporating a different number of phase masks into the system. Fig.5 shows the reconstructed images obtained from systems with different number of phase masks incorporated to them. It can be observed that the more the optical channels incorporated with a phase mask, the better the quality of the reconstructed image is. This shows a trade off between the number of phase 


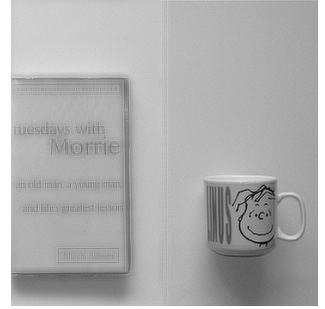

(a) One phase mask

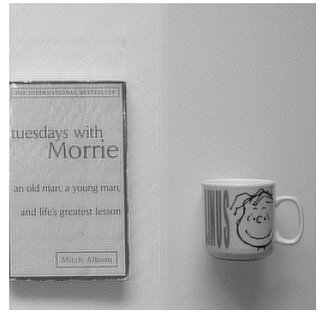

(c) Three phase masks

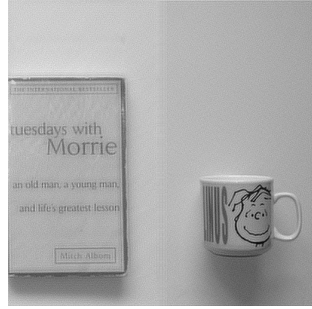

(b) Two phase masks

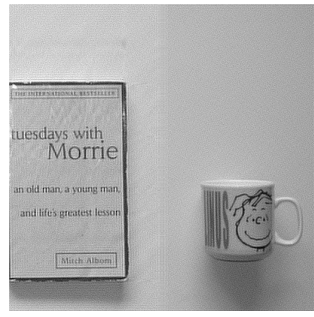

(d) Four phase masks

\section{Figure 5. Reconstructed images with differ- ent numbers of phase masks incorporated.}

masks (which means cost) and the image quality. It should be noted that if only some of the optical channels are incorporated with phase masks, there is an issue regarding which channels the phase masks should be placed. In our case of four optical channels, only the case for two phase masks should be of concern since any ways of positioning of the phase masks are symmetric for other cases. In the case for two phase masks inserted into two out of four of the optical channels, two ways are possible: i) placing in side-by-side channels, or ii) placing in diagonal channels. It can be observed in Fig.6 that the system with phase masks placed in diagonal channels gives an image with better visual quality than that produced by the system with phase masks placed in side-by-side channels.

\section{Conclusion}

We have established and investigated a compound-eye imaging system with super-resolution reconstruction. The feasibility of the incorporation of cubic phase masks in such a system with Wiener filter restoration to extend the depth of field has been verified. Simulation results has demonstrated the effects of the number and positioning of phase masks on the visual quality of the reconstructed image.

\section{References}

[1] N. K. Bose and K. J. Boo. High-resolution image reconstruction with multisensors. International Journal of Imaging

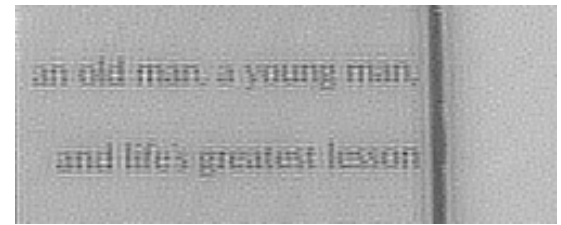

(a) Phase masks placed in side-by-side channels.

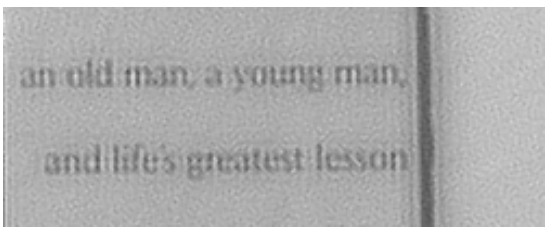

(b) Phase masks placed in diagonal channels.

\section{Figure 6. Reconstructed images with two phase masks incorporated.}

Systems and Technology, 9(4):294-304, December 1998.

[2] E. R. Dowski, Jr., and W. T. Cathey. Extended depth of field through wave-front coding. Applied Optics, 34(11):18591866, April 1995.

[3] J. Duparré, P. Schreiber, P. Dannberg, T. Scharf, P. Pelli, R. Völkel, H.-P. Herzig, and A. Bräuer. Artificial compound eyes - different concepts and their application to ultra flat image acquisition sensors. In MOEMS and Miniaturized Systems IV, volume 5346 of Proceedings of the SPIE, pages 89-100, January 2004.

[4] J. Duparré, P. Schreiber, A. Matthes, E. Pshenay-Severin, A. Bräuer, and A. Tünnermann. Microoptical telescope compound eye. Optics Express, 13(3):889-903, February 2005.

[5] M. Elad and A. Feuer. Restoration of a single superresolution image from several blurred, noisy, and undersampled measured images. IEEE Transactions on Image Processing, 6(12):1646-1658, December 1997.

[6] J. W. Goodman. Introduction to Fourier Optics. McGrawHill, 2nd edition, 1996.

[7] Y. Kitamura, R. Showgenji, K. Yamada, S. Miyatake, M. Miyamoto, T. Morimoto, Y. Masaki, N. Kondou, D. Miyazaki, and J. Tanida. Reconstruction of a highresoloution image on a compound-eye image-capturing system. Applied Optics, 43(8):1719-1727, March 2004.

[8] M. K. Ng and N. K. Bose. Mathematical analysis of superresolution methodology. IEEE Signal Processing Magazine, 20(3):62-74, May 2003.

[9] M. K. Ng, R. H. Chan, T. F. Chan, and A. M. Yip. Cosine transform preconditioners for high resolution image reconstruction. Linear Algebra and Its Applications, 316(13):89-104, September 2000.

[10] J. Tanida, T. Kumagai, K. Yamada, S. Miyatake, K. Ishida, T. Morimoto, N. Kondou, D. Miyazaki, and Y. Ichioka. Thin observation module by bound optics (TOMBO): Concept and experimental verification. Applied Optics, 40(11):18061813, April 2001. 